\title{
Suivre le pas d'Ismène : une alternative à la temporalité tragique
}

Préférant le roman à la tragédie pour son Antigone, Henry Bauchau se distingue de la plupart des (r)écritures ${ }^{\mathbf{1}}$ de l'Antigonè de Sophocle, majoritairement théâtrales. Le changement générique instaure une nouvelle dynamique narrative et provoque un ralentissement général de la diégèse. Le cheminement du personnage, l'importance accordée au "vécu entre les faits » (EE, p. 59) et aux évènements du quotidien constituent le véritable objet du roman. Inversant la conception narratologique traditionnelle, le roman de Bauchau fait ainsi des temps morts du mythe ses temps forts. Il met au centre de son propos les instants en apparence faibles du récit mais en réalité chargés de tout l'« aspect concret et sensoriel de la vie, qui en fait toute la densité vécue "². Toutefois, le statut de (r)écriture de son Antigone implique une autre temporalité qui sourd : celle de la trame antique qui demeure en filigrane et joue avec les attentes du lecteur pour mieux les déjouer ensuite. Cette tension entre temps du texte et temps de l'intertexte instaure ainsi une impression sporadique de coprésence $^{3}$. Le roman accentue cet effet en mettant en place de nouvelles scènes d'énonciation qui complètent la diégèse principale.

Nous étudierons tout d'abord ce phénomène de coprésence et les différents régimes de temporalité qu'il convoque. Cette polyphonie discursive et intertextuelle trouve son paroxysme dans la transfiguration finale de l'Antigone d'Io. S'ouvre alors un nouveau lieu d'expression pour l'héroïne : le théâtre « où l'action et la parole, [...] échapp[ent] aux servitudes de l'actualité, à son perpétuel glissement dans l'oubli ou la banalité " $^{4}$. Tout au long du roman, la fusion de la voix d'Antigone et d'Io est en quelque sorte annoncée par les passages où l'héroïne délègue l'énonciation, notamment à sa sœur Ismène. Nous proposons de montrer dans cette étude le rythme particulier qu'Ismène imprime à son discours dans le roman et plus particulièrement dans le chapitre intitulé "Le monologue d'Ismène ». Comment l'irruption de ce nouveau tempo, associé à un timbre de voix propre à Ismène, participent-ils à la construction du sens pour cette Antigone moderne, résolument dialogique?

1 La notion de (r)écriture développée par Ute Heidmann permet de souligner la singularité d'une démarche dont l'objet d'étude "n'est pas le phénomène du mythe "en tant que tel", mais son écriture, comme une forme de représentation particulière avec ses lois propres ". (Ute Heidmann, "Comment comparer les (r)écritures anciennes et modernes des mythes grecs? Propositions pour une méthode d'analyse (inter)textuelle et différentielle ", dans Sylvie Parizet (dir.), Mythe et littérature, Paris, Société Française de Littérature générale et comparée, "Poétiques comparatistes ", 2008, pp. 143-160, p. 144 pour la citation). 2 Myriam Watthee-Delmotte, Henry Bauchau, Edipe sur la route. Un livre, une ouvre, Bruxelles, Labor, 1994, p. 99.

3 Gérard Genette, Palimpsestes. La littérature au second degré, Paris, Seuil, 1982, p. 8.

4 Henry Bauchau, "Au sujet d'Antigone ", dans Alternatives théâtrales n 56, décembre 1997, p. 4. 


\section{«La vie n'est plus tout à fait la vie mais une sorte d'attente, de ciel gris et de mort en suspens 》s}

À la différence de son modèle tragique soumis aux contraintes de sa propre performance théâtrale ${ }^{6}$, Bauchau choisit pour sa (r)écriture un genre qui " a le temps ", une forme qui lui permet de "développe[r], prolonge[r], détaille[r], analyse[r] sans être limité par rien d'autre que son souci de faire sens ${ }^{7}$ : le roman. La signifiance de l'univers poétique de Bauchau ne se construit plus sur la succession de hauts faits mais tourne autour de la vie quotidienne d'Antigone, une option permise par le genre romanesque dont la modernité s'attache à faire une forme qui peut se développer "à propos de l'infiniment petit, de l'absolument dérisoire " $\mathbf{8}$. Se crée dès lors une tension entre le choix d'une héroïne mythique, inscrite dans une tradition tragique - et plus largement dramatique - et son traitement narratif résolument moderne. Dans son Antigone, à l'instar de son Edipe, c'est "l'espace intermédiaire, resté jusqu'ici inexploré » que Bauchau [s']efforce "peut-être témérairement ", confie-t-il, " de parcourir "?.

Les chapitres IV à XVII qui composent le corps du roman, constituent une immense amplification par rapport à l'Antigonè de Sophocle à laquelle on a l'habitude de le comparer. Il faut pour les comprendre les rapprocher également des Sept contre Thèbes d'Eschyle, tragédie centrée sur l'affrontement entre Étéocle et Poylnice. La (r)écriture de Bauchau explore les causes de cette guerre civile (mais aussi les chemins d'une résolution possible du conflit) jusqu'à l'assaut final, pour exposer enfin la condamnation d'Antigone. L'œuvre noue donc, bout à bout, la trame de deux tragédies, tandis que le roman familial des Labdacides sert de châ̂non causal aux deux séquences narratives. Elle délaisse le conflit entre Antigone et Créon (que Sophocle aurait déjà " donné à voir et à entendre ", selon l'auteur, "de façon insurpassable „10) et se centre sur l'affrontement d'Étéocle contre Polynice, en prenant soin d'en situer la cause dans la petite enfance. L'imaginaire guerrier mis en place dans ces chapitres emprunte au souffle tragique et épique de la pièce d'Eschyle. Il rappelle surtout, à l'instar de Duras, combien « la banalité est frappante

\footnotetext{
5 A, p. 199.

6 La pièce de Sophocle se déroule dans l'espace d'une seule journée. Elle débute à l'aube. Puis, le garde découvre Antigone honorant le corps de Polynice "à l'heure où le disque du soleil atteint le

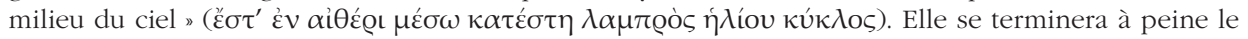

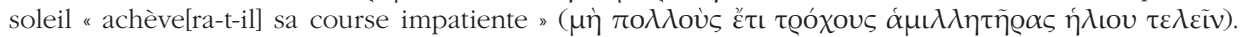
Le rapport entre le nombre et l'intensité des évènements, corrélée à la durée fictive de l'action, crée un rythme très soutenu. Cela répond à la notion de "tension tragique ", où la rapidité d'enchaînement des coups du sort augmente l'émotion du spectateur, tout en symbolisant l'inexorabilité du destin qui s'abat sur le héros (Sophocle, Antigone, traduit du grec par Paul Mazon, Paris, Les Belles Lettres, 2002, v. 415-416, 1064-65).

7 Nathalie Piégay-Gros, Le Roman, Paris, Flammarion, "GF-Corpus », 2005, p. 29.

8 Ibid., p. 15.

9 Jean Robaey, "Fonction et fonctionnement du mythe classique : de Sophocle à Bauchau ", dans Anna Soncini Fratta (dir.), Henry Bauchau. Un écrivain, une oeuvre, Bologne, CLUEB, 1993, p. 285.

10 Henry Bauchau, "Au sujet d'Antigone ", art. cit., p. 4.
} 
parfois "11. Loin des assauts in medias res des Sept contre Thèbes, c'est la durée du combat qui, chez Bauchau, fait horreur. Le texte l'explore, scandé par des expressions temporelles qui marquent la répétition comme "le lendemain ", fréquemment utilisé en tête des alinéas, ou " chaque jour ", repères qui laissent le lecteur dans un magma temporel imprécis. Le parallélisme de certaines de ces constructions augmente encore l'impression de pesante récurrence : "Les malades arrivent, ils arrivent chaque jour plus nombreux "(A, p. 174) ${ }^{\mathbf{1 2}}$. Alors que le malheur monte crescendo sur la ville, l'espérance d'Antigone, ses aspirations à une vie ordinaire s'amenuisent : "plongée dans la part malheureuse et souffrante de Thèbes, si occupée par les angoisses de ceux dont [elle a] la charge ", Antigone "n'arrive plus à imaginer un autre monde ni une autre vie " (A, p. 199). Facteur de résilience chez l'écrivain, la pratique de l'art devient impossible, comme le fait remarquer Dirkos : «- C'est mon atelier... / — Tu n'en auras plus besoin. [...] la guerre, la misère vont prendre toute ta vie, Antigone " (Ad, p. 179). Durant cette période, le roman explore la manière dont Antigone peut, "dans le champ du malheur, planter une objection " (JA, p. 138). Par la création d'un dispensaire, elle recrée aux côtés de sa sœur des conditions de vie temporairement acceptables, tout en prenant part à la souffrance de la cité.

\section{Une lente fuite en avant}

Malgré un ralentissement général de la diégèse, le récit conserve du modèle antique, selon l'auteur, le caractère de "course ", dans laquelle la jeune fille se précipite " poussée par ce qu'elle est " (JA, p. 341). Forte de toute une tradition littéraire, Antigone est appelée dès l'incipit à suivre le " chemin du rouge " tracé par la querelle de ses frères. Elle sera bientôt submergée par leur folie (à moins qu'à l'instar des adversaires de la fresque, ceux-ci trouvent un moyen de se confronter sans se détruire). La récurrence d'images rompant avec la continuité du récit suggère le dénouement funeste (qui sera nuancé par la transfiguration de l'Antigone d'Io), comme dans cet exemple : "Ismène et parfois Étéocle veulent m'expliquer ces événements mais je n'entends rien d'autre que le bruit d'une énorme trappe qui nous précipite lentement dans l'abîme "(A, p. 204). Cette récurrence crée une impression de lente fuite en avant, appuyée par l'inscription de l'hérö̈ne dans la tradition littéraire et étayée par des indices symbolisant l'affrontement final par des métaphores s'y rapportant, des visions de l'héroïne, etc. Le lecteur comprend toutefois qu'il ne s'agit pas, comme dans la tragédie athénienne, de malédiction ou de destinée. La trajectoire des personnages émerge de leur parcours personnel et ce qu'il leur a permis de devenir. Ils sont « natif[s] de [leurs] ruines surgissantes " (PC,

11 Marguerite Duras, Hiroshima mon amour, Paris, Gallimard, 1960, p. 140. Cette préoccupation de l'impact de la guerre sur les plus faibles semble déjà annoncée par le chour de la tragédie d'Eschyle composé des femmes thébaines qui attendent dans le gynécée l’issue des combats.

12 Pour les autres occurrences, "le lendemain ": A, p. 50, 59, 69, 81, 95, 127, 168, 170, 178, 180 ; " chaque jour": p. 174, 176. Elles sont complétées par les expressions suivantes : "dans les jours qui suivent " (A, p. .67), "pendant quelques jours " (A, p. 73), "quelques jours plus tard » (A, p. 74), " pendant de nombreux jours " (A, p. 151), "après quelques jours " (A, p. 168), "les jours s'écoulent " (A, p. 75). 
p. 151), telle Antigone qui agit en adéquation avec la vérité intérieure découverte auprès de son père :

"En retournant à Thèbes on dirait que tu obéis à un ordre.

- Il n'y a pas d'ordre, Clios, mais un amour, une compassion pour Thèbes et pour mes frères auxquels je dois obéir. "

Il gronde : "Obéir, qu'est ce que cela veut dire obéir ? Obéir à quoi ? "

Je suis surprise qu'il ne comprenne pas ce qui est si évident, surtout dans sa montagne : "Obéir comme une plante qui sort de terre, comme un ruisseau qui s'écoule. " (A, p. 36)

L'impératif moral ne prend pas ici la forme d'une requête extérieure (il n'y a pas d'ordre), ni ne répond à une malédiction comme dans la tragédie. Désormais intériorisé, il n'est plus contrainte, mais s'avère conforme à la nature profonde de celui qui s'y soumet. Il revêt alors un caractère de simplicité (ce qui est si évident), puisque le sujet est invité à agir en adéquation avec son être intime. Simple lorsqu'on l'a découverte, cette vérité s'apparente à la petite fille " obscure à ellemême ", évoquée par Antigone, qui l'appelle à Thèbes " avec une voix faible, mais irrésistible " (A, p. 36). Fruit d'une longue initiation, elle ne connaît qu'une règle, celle de l'empathie, dans son sens le plus large : il s'agit de s'éprouver face à l'autre mais aussi face à son autre par l'exploration des forces de l'inconscient. Si Antigone s'est construite dans le cheminement de la route, elle semble désormais appelée à s'éprouver dans la société. Il s'agit d'un écart conséquent par rapport à la pièce de Sophocle qui met au contraire en scène l'échec de l'insertion de l'hérö̈ne dans la cité ${ }^{\mathbf{1 3}}$. La narration de l'occupation de Thèbes, qui trouve des échos dans la biographie de l'auteur, illustre donc la manière dont " le possible, avec la guerre, ne cesse de rétrécir » (A, p. 199).

La construction de cet espace diégétique, non héroïque, trace d'un même trait les conditions pour l'héroïne d'un potentiel être ensemble. Sur cette nouvelle scène de la quotidienneté et de l'appartenance à la communauté, Antigone devient une figure évolutive et relationnelle, " une matière vivante, un personnage riche en complexité identitaire "flexible" qui s'organise et se structure dans un parcours existentiel et dans un monde relationnel ", comme le souligne Emilia Surmonte ${ }^{\mathbf{1 4}}$.

13 Selon cette interprétation de Sophie Klimis, Antigone chez Sophocle est représentée comme une figure a-politique. En effet, si l'on tient compte de l'ensemble de la performance, la tragédie oppose non plus deux personnages, Antigone et Créon, mais illustre plutôt, grâce à eux, les facettes d'une même bybris. La thèse est également défendue par Cornélius Castoriadis : "le thème central de l'Antigone, c'est l'bybris, la démesure qui réside dans le "penser seul" (monos phronein) " (Sophie Klimis, "Antigone et Créon à la lumière du "terrifiant/extraordinaire" de l'humanité tragique ", dans Lambros Couloubaritsis et François Ost (dir.), Antigone et la résistance civile, Bruxelles, Ousia, 2004, p. 63-102, ici p. 65). Ce travers fait d'Antigone, comme de Créon, des personnages résolument seuls. Je voudrais montrer que dans les textes de Bauchau, il s'agit au contraire de faire grandir au contact de l'autre, de limer tout son être à celui d'autrui.

14 Emilia Surmonte se réfère ici aux recherches de Giuseppe Bottiroli concernant la modalité de construction des personnages et la distinction " entre identités rigides et identités flexibles : les unes se définissent en fonction des propriétés qui leur sont attribuées et les autres en fonction des relations intersubjectives et actionnelles qui les construisent et les modèlent. " (cité par Emilia Surmonte dans 


\section{«Se donner du temps, c'est le grand don qu'on doit se faire $\gg 15$}

Grâce à l'extension de la durée diégétique, la possibilité d'une vie normale pour l'hérö̈ne semble envisagée dans la première partie du récit. Cette période qui participe à redéfinir le rythme de l'action est placée sous l'égide d'Ismène. Dans l'Antigonè de Sophocle, ce procédé d'évocation puis de révocation du bonheur participe au ressort tragique. Il repose en grande partie sur le personnage d'Ismène qui évoque pour sa sour un autre mode de vie possible, fondé dans ce contexte sur la représentation d'un éthos féminin ${ }^{\mathbf{1 6}}$. Ismène chez Bauchau se fait quant à elle l'écho de préoccupations modernes : "Je suis la seule de la famille à penser au bonheur, à mon bonheur à moi " (A, p. 152) ${ }^{\mathbf{1 7}}$. Cette quête identitaire d'Ismène (marquée par le caractère pléonastique de l'expression " mon bonheur à moi ") la place en contre-pied des membres de sa famille, un bonheur qu'elle tente de "préserver des ambitions d'Etéocle, du rire souverain de Polynice, des combinaisons de Créon et de l'extravagante étendue de ton âme, Antigone "(A, p. 152). En disant non à " un certain héritage auquel Antigone n'a pas su/voulu se soustraire „18, Ismène offre à sa sœur un temps de répit, comme celle-ci le comprendra plus tard:

Depuis mon retour à Thèbes, c'est toi qui as été la grande sœur, c'est toi qui sans cesse m'as protégée de ton affection et de ta clairvoyance. Le peu que j'ai pu faire, c'est grâce à toi. Sans ta patience - et tes colères contre mes illusions - tout aurait tourné plus mal encore et plus vite. Au lieu de pouvoir dire non à Créon comme tu m'en as donné le temps et la force, je serais morte depuis longtemps. (A, p. 324, nous soulignons)

Ce temps supplémentaire semble ainsi intimement lié à la figure d'Ismène (comme le montre la répétition de l'expression " c'est toi " et de sa variante " c'est grâce à toi " qui mettent syntaxiquement en exergue la figure du $t u$ ). Répondant au texte antique, le roman crée une nouvelle figure d'Ismène qui semble tracer à sa sœur la voix du bonheur. Alors que l'amour n'est pas considéré dans l'Antiquité comme un ressort dramatique, le roman développe l'attachement d'Antigone pour Hémon d'une façon explicite ${ }^{\mathbf{1 9}}$. C'est littéralement à la suite d'Ismène qu'Antigone

Antigone, la Sphinx d'Henry Bauchau. Les enjeux d'une création, Bruxelles, Peter Lang, "Documents pour l'histoire des francophonies ", 2011, p. 36).

15 JJ, p. 303.

16 Sophie Klimis, art. cit., p. 86.

17 Une citation qui semble faire écho aux mots prononcés par l'Antigone antique, comme à l'encontre de sa sœur : "Voyez, [...] la seule qui survive des filles de vos rois " ( $\lambda \varepsilon \dot{v} \sigma \sigma \varepsilon \tau \varepsilon[. ..] \tau \dot{\eta} v \beta \alpha \sigma \iota \lambda \varepsilon \iota \delta \tilde{\omega} v$

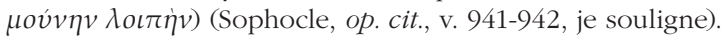

18 Christiane Chaulet-Achour, "Le personnage d'Antigone : une approche poétique de l'androgynie ", dans Catherine Mayaux et Myriam Watthee-Delmotte (dir.), Henry Bauchau. Écrire pour habiter le monde, Vincennes, Presses universitaires de Vincennes, 2009, p. 227-238, ici p. 237.

19 Ce motif prend sa source dans l'interrogation du romancier face à la trame antique : "Tout cela n'est pas encore clair, pourtant entre Antigone et Hémon il y a un amour. Sans cela, comment expliquer le suicide d'Hémon ?" (JA, p. 176.) Dans la tragédie de Sophocle au contraire, nubile mais ignorant le mariage, Antigone vit dans un hors-temps contraire à l'ordre social de son époque (voir, à ce sujet, Florence Dupont, L'Insignifiance tragique, Paris, Gallimard, 2001). De toute la pièce, elle ne prononcera le nom de son fiancé, Hémon, à l'exception du kommos, ce chant de deuil chantée à 
tombe amoureuse d'Hémon : "Je vois que tu as déjà recruté le bel Hémon. Il m’a aimé avant toi [...]»(A, p. 62). Antigone semble alors s'inscrire, à sa mesure du moins, dans le sillage du sensualisme d'Ismène (dont le corps " tout en courbes douces orientées vers le plaisir " capte les regards des hommes par ses " mouvements onduleux ", A, p. 49 et 226). L'hérö̈ne peut alors dire : " je suis contente d'avoir cherché à lui plaire, comme n'importe quelle femme, à un homme qui lui plaît " (A, p. 141). Antigone n'est plus " insensible au désir des hommes, ni au sien, mais elle est appelée à autre chose " (JA, p. 397). Le motif de la virginité est ainsi conservé mais " plus valorisé ", précise l'auteur. Il répond désormais aux différentes tensions génériques en œuvre dans le récit et à leurs différents tempos. Se succèdent ainsi les passages où Antigone est " pleinement femme " et ceux " épiques ", où elle se fait "plutôt androgyne " (JA, p. 496). Si Antigone doit comme Jeanne d'Arc, " assumer l'acte héroïque " (JA, p. 94), Ismène, symbole de cette sagesse pratique chère aux Grecs (sophrosunè), devient le porte-parole de préoccupations concrètes. Le récit rend compte de la rencontre de ces deux manières d'être au monde, ce qui nuance et adoucit la représentation d'Antigone :

[Ismène] voit bien que parfois je suis ailleurs et me dit un jour : "Antigone, tu es en train de devenir une voyante, comme notre père. " / Elle éclate de rire. / "Tu as mieux à faire!» / Ah ! que ce rire me fait du bien, je lui saute au cou et je ris avec elle : / "Heureusement que je t'ai Ismène. Grâce à toi je peux vivre mes visions et ne pas devenir idiote!» (A, p. 208-209)

Cette figuration particulière d'Ismène, en germe chez Sophocle, passe chez le romancier par l'invention de "sa propre vie ", un espace intime préservé dont elle interdit l'accès à "sa redoutable famille " (A, p. 48), comme au lecteur et qui trouve son apogée dans la maternité d'Ismène. Protagoniste du quotidien, dans laquelle elle entraîne sa sœur, Ismène n'est pourtant pas dépourvue d'un certain héroïsme, comme le prouve le rôle qu'elle tient lors de l'assaut de Thèbes. Antigone est en effet invitée à entendre "la leçon d'Ismène " que Christiane Chaulet-Achour décrit en ces termes :

[L']héroïsme est plus facile à tenir que la résistance au quotidien qui accepte les compromissions pour assurer la survie. C'est bien une énergie de chaque jour qu'Ismène déploie [...]. Ismène vit, elle est apte au bonheur, elle fait les gestes qu'il faut quand il faut, elle dit ce qu'il faut entendre [.... ${ }^{20}$

Le timbre d'Ismène se détache du récit, grâce à l'utilisation de modalités énonciatives particulières et étrangères à Antigone. Sa parole prend avant tout le visage d'une interruption dans le flot linéaire des paroles et pensées d'Antigone qui composent le roman. Ses éclats de rire récurrents brisent la continuité des récits de sa sœur ("un rire crispé d'Ismène me répond ") ; son propos contraste souvent par sa franchise ("Arrêter tous ces mâles, Antigone, c'est comme si tu croyais que leurs sexes vont cesser de se dresser pour nous »). Sa gestuelle et ses mouvements

elle-même pour regretter d'une façon très abstraite son hyménée. Ismène, au contraire, rappelle les fiançailles au vers 568 pour témoigner de la légitimité de ce lien.

20 Christiane Chaulet-Achour, art.cit., p. 236. 
brusques ("Ismène s'écarte vivement de moi, elle me fait face, ses yeux brillent de colère »), ses sarcasmes (" quels voisins, heureusement que ce grand jardin te préserve de leurs odeurs » $\mathbf{2 1}$ ) sont autant de marques de décrochage par rapport à la source principale d'énonciation.

\section{«Une sororité active »22, un contre-modèle pour les frères ennemis?}

Dans cette (r)écriture moderne, l'enjeu politique a cédé le pas à l'éthique. Il s'agit de mettre en place une représentation du monde avant tout dialogique. Figures résolument évolutives à l'inverse des frères, les deux sœurs se transforment au contact l'une de l'autre. Une leçon que l'auteur semble prendre à son compte : alors qu'il concède dans son Journal que ce qui réellement lui importe, "c'est ce qui se passe dans l'esprit d'Antigone ", il prend néanmoins soin de révéler "le revers de l'aventure initiatique d'Antigone en montrant ce qu'elle a coûté à Ismène " (JA, p. 310). Cela se traduit par un traitement particulier de la relation entre Ismène et Antigone, dans laquelle transparaît la notion d'échange (présentée dans l'incipit comme un possible élément de résolution de conflit entre Étéocle et Polynice). Le lecteur assiste à de continuelles frictions entre les sœurs, une représentation qui illustre la complexité des relations familiales et leur ambiguité : "— Tu me détestes, Ismène ? / — Naturellement je te déteste, je te déteste presque autant que je t'aime " (A, p. 53). L'espace diégétique interstitiel habité par les deux sœurs, dévoile néanmoins une volonté de cheminer ensemble par-delà les différends et les différences, marquée par la récurrence de la marche conjointe : "Je n'ai pas envie de parler mais de sentir nos deux corps marcher d'un même pas comme nous l'avons fait si longtemps " ou encore " Nous avons besoin d'être ensemble et de marcher côte à côte sans parler » (A, p. 47 et 62, respectivement). Dans ces moments, le pas de la cadette, "gracieux [et] entouré des sourires de tous ceux qui la croisent " rejoint alors, sans l'épouser complètement, celui " trop masculin " de l'aînée, " qui est devenu le [s]ien et qui doit déplaire à Créon " (A, p. 76). Chacune accorde ainsi sa cadence à celle de l'autre et rappelle ainsi "l'harmonie discordante " déjà à l’œuvre dans la tragédie athénienne, selon Sophie Klimis ${ }^{\mathbf{2 3}}$. Ces moments de partage,

21 A, p. 89, 101 et 61, passim, pour les citations entre parenthèses.

22 J'emprunte l'expression à Christiane Chaulet-Achour, art. cit., p. 237. Dans cette partie, je me réfère en outre à l'article de Véronique Jago-Antoine, "La transposition du geste pictural dans le cycle oedipien d'Henry Bauchau ", dans Marc Quaghebeur (dir.), Les constellations impérieuses d'Henry Bauchau. Colloque de Cerisy 21-31 juillet 2001, Bruxelles, AML Éditions / Éditions Labor, 2003, p. 413-436.

23 Cette "harmonie discordante " est une modulation de l'expression " harmonie oscillante ", traduction que donne Cornelius Castoriadis à l'expression d'Héraclite "palintropos harmoniè " (fragment B51). Traduisant plutôt ce syntagme par " harmonie discordante ", Sophie Klimis l'utilise pour appréhender cette dynamique particulière à l'œuvre dans les tragédies athéniennes. À propos du fragment d'Héraclite d'où est tiré cette expression, la philosophe précise : Castoriadis le cite dans ses séminaires, en le glosant de la manière suivante : "Les hommes ne comprennent pas comment ce qui s'oppose à soi-même s'accorde avec soi-même, une harmonie oscillante qui a deux vecteurs opposés, comme celle de l'arc et de la lyre (pour tendre un arc, il faut en effet que jouent deux forces opposées). C'est ce que les hommes ne comprennent pas lorsqu'ils considèrent les contraires comme des entités fixes et séparées, et la contrariété comme négative et destructrice ; alors que (fr. 8) ce qui s'oppose 
tout comme les épisodes de bain qui interviennent à des instants stratégiques du récit (au retour d'Antigone à Thèbes, puis au moment de prendre la décision d'enterrer Polynice) revêtent alors un caractère rituel. Ils anticipent la toilette funéraire de Polynice, un geste auquel Antigone associe symboliquement Ismène, contrairement à son homonyme antique ${ }^{\mathbf{2 4}}$. Ces passages sont le lieu d'une réminiscence partagée : «Un mode de présences disparues et de souvenir remonte en nous, tout l'univers rieur et déchirant d'avant la séparation " (A, p. 49). Il s'y dessine l'esquisse d'une réconciliation comme dans cet extrait où la formulation proche du discours direct marque la vive émotion d'Antigone : "Je me trouble, je ne peux continuer, à quelle profondeur, avec quelle force nous sommes restées sœurs malgré la séparation et les années »(A, p. 48). Par la confrontation et le dialogue, les deux sœurs sans cesse soutenues par l'affection qu'elles se portent, trouvent une communication qui leur est propre. Par la convocation de tous les sens (" un bras léger se glisse sous le mien, je reconnais un parfum » - A, p. 47), les sœurs parviennent à réactiver "l'anneau d'émotions et de souvenirs qui [les] unit " (A, p. 71) 25. Par-delà les tensions, Ismène et Antigone déjouent les pièges de la préférence, cette comparaison aliénante qui semble au cœur de l'antagonisme fraternel et dans laquelle "l'un [reste] le modèle et l'autre la copie à jamais incapable de l'égaler " ${ }^{\mathbf{2 6}}$. Les sœurs peuvent dès lors évoluer côte à côte, chacune dans leurs différences et mettre en place une sororité active. Cette dernière atteint son climax dans l'épisode de création conjointe mais s'avère selon nous opérante dès les retrouvailles à Thèbes et jusqu'à leur décision conjointe d'enterrer Polynice. Cette complicité se construit à travers le projet commun du dispensaire, puis des statues de Jocaste.

s'accorde à soi, la plus belle harmonie est celle qui est produite par des entités en conflit, et tout advient selon l'éris discorde et dispute. " (Sophie Klimis, "La muscalité sémantique du penser-poème grec. Pour une eidétique du prattein-poein dans le langage ", dans Philippe Caumières, Sophie Klimis et Laurent Van Eynde [dir.], Cahiers Castoriadis, n 5, Bruxelles, Publications des Facultés Universitaires SaintLouis, p 173-242, ici : p. 188-189, nous soulignons).

24 Il s'agit d'un élément qui répond au texte antique. Dans la tragédie, Antigone demande dans un premier temps l'aide de sa sœur pour enterrer Polynice dans une formulation qui insiste sur le partage du

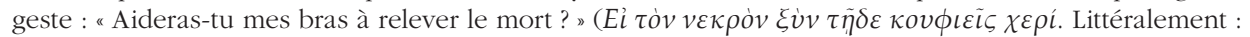
"si tu soulèves le mort avec cette main ": Sophocle, op. cit., v. 43). Elle refusera plus tard d'en partager la retombée symbolique comme le souhaiterait pourtant Ismène. Dans le roman, c'est l'inverse qui se passe. La décision est commune : "— Son corps... Pourrir ! Jamais je ne permettrai ça... ! Ismène non plus ». Comme Ismène ne peut participer à l'ensevelissement à cause de sa grossesse, sa sœur l'associe symboliquement au rituel : c'est alors qu' avec vénération, au nom d'Ismène, d'Étéocle et de nos parents ", Antigone "pose le masque d'argent sur sa figure autrefois si fière " (A, p. 288 et 296).

25 Le caractère dialogique du roman ne peut selon nous se comprendre sans mesurer la différence qui le sépare de la tragédie. Dans celle-ci, dès le prologue, Antigone presse sa sœur à trois reprises de l'aider à enterrer Polynice (v. 34, 41, 43). Face à son refus, Antigone répond alors : "Sois tranquille, je ne te demande plus rien - et si même tu voulais plus tard agir, je n'aurais pas la moindre joie à te sentir à mes

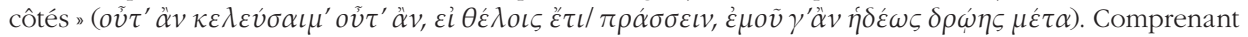
que sa sœur désapprouve son projet, Antigone la considère dès lors comme une étrangère. Ismène, au contraire, demeure pleine d'empathie à son égard et déclare : "À ton gré, pars ; mais sache, en partant, que tu restes, en dépit de ta folie, justement chère à ceux qui te sont chers. " ( $\dot{\alpha} \lambda \lambda^{\prime} \varepsilon \dot{\varepsilon} \delta o \kappa \varepsilon \tilde{\imath} \sigma o l, \sigma \tau \varepsilon \tilde{\varepsilon} \chi \varepsilon$.

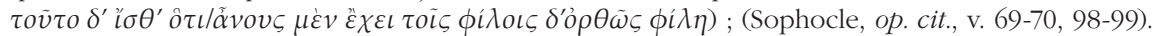

26 Cette thématique de l'identification aliénante est déjà à l'œuvre dans la tragédie de Sophocle. Comme le montre Nicole Loraux, dès les premiers vers, c'est à "identifier sa sœur comme une autre soi-même que vise Antigone " (Nicole Loraux, "La main d'Antigone ", dans Mètis. Anthropologie des mondes grecs anciens, $\mathrm{n}^{\circ} 1,1986$, p. 165-196, ici p. 172). 
La temporalité interstitielle offerte par le roman constitue une alternative à la solitude d'Antigone. Durant celle-ci, Ismène, protagoniste principale, ménage à sa sœur une trêve (à l'image de celle de leurs jeux d'enfants) dans sa sombre trajectoire.

\section{Les deux faces de Jocaste}

Dans le chapitre intitulé "Les sculptures ", Antigone fait face à la demande formulée par Étéocle de créer deux bas-reliefs de Jocaste : un pour Polynice, un pour lui. Ceux-ci auraient une vertu incantatoire et permettraient d'exorciser la ville de Thèbes de la figure de la reine morte. Ils doivent mettre de l'avant « la ressemblance et l'inépuisable différence entre ces deux Jocaste qui ont conditionné " (A, p. 93) la vie des jumeaux. La (r)écriture moderne opère sur ce point un déplacement fondamental face à la tradition antique. La guerre fratricide n'est plus simple lutte de pouvoir, comme l'écrivain le comprend dans un rêve : "la rivalité des deux frères au sujet de Thèbes vient de ce que Thèbes est la terre de la mère " (JA, p. 249). Loin de la symbolique traditionnelle du portrait de famille qui représente l'unité d'une fratrie, le double portrait de Jocaste dévoile la généalogie trouble des Labdacides et le rapport différencié de la mère à ses fils. Par le biais des statues, Polynice doit ressentir l'injustice commise et comprendre qu'étant roi par nature (le don lui ayant été conféré implicitement par la préférence de Jocaste), il n'a nul besoin d'en étreindre les attributs réels. Suppléante de la parole maternelle, Antigone devient la réparatrice des fautes de Jocaste, celle en qui les jumeaux deviennent égaux. Le choix du basrelief, ce support où le sujet représenté ne se détache que faiblement de son fonds et reste engagé à mi-corps dans la matière, peut être mis en lien avec l'enlisement du sujet dans l'univers familial. La volumétrie de la sculpture donne ainsi corps à l'injustice, en esquissant les contours du poids que ce désamour a constitué pour Étéocle.

\section{Le monologue d'Ismène}

Devant la difficulté de la tâche qui lui est confiée, Antigone confesse son impuissance à la mener à bien toute seule. Sur les conseils d'Étéocle, elle s'adresse à Ismène :

"J'ai besoin que nous parlions, Ismène.

- De quoi, de qui ?

De notre mère, de nos frères, de nous deux. J'ai besoin de parler avec toi, rien qu'avec toi. " [...]

"Je vois bien ce que tu veux, tu dois pour tes sculptures évoquer notre mère et les jumeaux. Ce sera douloureux et tu vas retomber dans tes pleurnicheries. Tu les détestes, bien sûr, mais aussi tu les aimes, et pour sculpter tu dois avoir les yeux clairs. » (A, p. 101)

Dans le chapitre qui porte son nom, Ismène opère un véritable tour de force, en imprimant aux propos qui suivent son ton. Répondant à l'injonction silencieuse d'Antigone, Ismène reformule la requête afin d'en rendre saillant le coût émotionnel : "Ce que tu veux c'est que ce soit moi, ta petite sœur, qui te parle d'eux et que j'accepte de pleurer à ta place pour que tu puisses travailler en paix [...] et que ce soit moi qui souffre "(A, p. 101). Après avoir redéfini les modalités de sa 
participation à la création des statues, elle se réapproprie l'espace énonciatif, en s'y assignant de nouvelles fonctions, comme dans cet extrait :

Puisque tu le veux, Antigone, je parle, je parle et tu te tais. Pourtant nous parlons puisque je me saisis de ton silence et parviens parfois à lui donner un sens. Je parle, je vais, je viens, je tourne autour de toi, je me fâche, j'éclate de rire, tandis que toi, assise devant ton établi, tu regardes sans fin ce que, pour te faire sortir de tes gonds, j'appelle tes bûches. (A, p. 103)

Si cette réplique débute avec le rappel de la requête d'Antigone (mise en relief par l'utilisation d'un vocatif), la protagoniste principale est ensuite invitée à se faire discrète par la triple présence de l'expression " je parle " ainsi que plus généralement l'omniprésence du " je " d'Ismène. Ce syntagme, à la connotation tautologique, est ici utilisé par Ismène pour occuper l'espace énonciatif, précédemment saturé dans le roman par la présence de sa sœur. Il prend dans ce contexte une valeur d'impératif, qu'il partage avec le " et tu te tais " adressé à Antigone. Dans ce dernier, la récurrence de la lettre " $t$ ", couplée à la brièveté des quatre monosyllabiques successivement utilisés, marque l'arrêt prononcé à l'encontre de la parole sororale. Les mots d'Ismène semblent au contraire rythmés par la répétition du " je parle " et comme entraînés vers la suite de l'échange par la succession de sons " $\mathrm{p}$ » qui lui font écho : ( je parle, je parle [...]. Pourtant nous parlons puisque je me saisis de ton silence et parviens parfois à lui donner un sens. Je parle ») Se saisissant de la causalité contenue dans le premier " puisque tu le veux ", Ismène l'inverse dans la phrase suivante. Prenant la parole sur demande de sa sœur, elle décide qu'il s'agira d'un dialogue tacite dont elle se fera l'interprète (" nous parlons puisque je me saisis de ton silence »). Dans la suite de l'extrait, le " je " d'Ismène, mobile et volubile, s'oppose à la figure du " tu ", silencieuse et immobile. Sa présence sature tant l'espace verbal que spatial, tous deux emplis de ses déplacements impromptus (" je vais, je viens, je tourne autour de toi ») et de ses mouvements d'humeur ("je me fâche, j'éclate de rire »). La répétition du pronom personnel, facultatif dans cette suite de juxtaposées, renforce l'omniprésence du " je ", tout comme la brièveté de ces propositions contrastent avec l'apparente passivité du "tu " ("assise devant ton établi, tu regardes sans fin »). Enfin, Ismène tourne en dérision l'entreprise artistique d'Antigone, renommant sarcastiquement l'ébauche des statues, "[l]es bûches", ou " ce morceau de bois crasseux " (A, p. 100).

Dans cet échange, Antigone est invitée à se faire discrète, passant du statut d'énonciatrice toute puissante, concédée par la focalisation interne du roman, à celui de co-énonciatrice. Ismène décide du temps imparti à leurs échanges, qui ne devrait pas empiéter sur sa propre vie. D'emblée, elle précise : "Quand le temps que j’ai décidé de consacrer à nos parleries est terminé, je m’éloigne " (A, p. 103). Elle n’y consacrera que "le temps qu'il faudra mais pas un instant de plus " (A, p. 101). Elle choisit une histoire parmi tous les récits possibles : "Aujourd'hui, je ne veux plus parler de notre vie d'autrefois. Je veux te parler d'CEdipe, de la vie d'CEdipe et pas seulement du père admirable et joueur de nos petites années " (A, p. 101). Elle s'approprie le discours (grâce à l'expression modalisée " je veux " qui répond à " je ne veux plus ») 
et ancre ainsi son récit dans le présent par l'usage des déictiques (le " aujourd'hui » qu'elle oppose à " autrefois »). Cette proximité entretenue par Ismène avec le moment d'énonciation reste constante tout au long du monologue, une attitude qui rappelle le " droit du présent ", face à l'héritage tragique dont Antigone semble la dépositaire.

Forte de cette nouvelle emprise sur le discours, Ismène décide de centrer son propos sur elle-même et évoque ce qu'elle a vécu comme une injustice : "La première année après ta fuite de Thèbes a été pour moi la plus dure, je me sentais seule, perdue dans ce palais où $m a$ mère était morte et où mon père et $m a$ sœur m'avaient abandonnée" (A, p. 104, je souligne). Au vu de la présence d'Antigone, l'utilisation de possessifs dénote (l'usage exigerait l'utilisation de "notre mère " et de "toi ma sœur, vous m'aviez abandonnée »). Ce procédé de mise à distance instaure l'exclusion temporaire d'Antigone de la situation de communication (celle-ci n'est plus considérée comme co-énonciatrice mais se retrouve dans la situation de non-personne). Par ces mots, Ismène oblige sa sœur et le lecteur à prendre conscience du revers de l'aventure initiatique d'Antigone, son abandon. Ces reproches sont toutefois prononcés selon une modalité discursive propre à ce personnage, où elle réaffirme les liens avec les êtres qui lui sont chers. Tout comme Antigone se rapproche de l'ouvre à faire par le contact du bois et par le souvenir plein d'émotion de sa mère, Ismène ressent le besoin de se réapproprier son outil, sa voix et sa langue, afin de s'affirmer en tant que sujet de son récit. Elle fait valoir sa connaissance de "l'CEdipe des autres " (A, p. 104), acquise auparavant par le dialogue. Par son emprise sur le moment d'énonciation en cours, Ismène se pose en sujet narrateur. "Dans ce processus de construction subjective qui opère une nouvelle mise en forme de l'expérience vécue ", Ismène engage " une part [d'elle]-même et de son rapport au monde ${ }^{27}$. Le personnage cherche une action et un discours en adéquation avec sa nature profonde, ce qui explique son premier mouvement d'incrédulité, alors qu'elle est associée à la tâche : "T'aider à quoi ? Je ne suis pas sculpteur, moi, je fais les plus belles tapisseries de Thèbes, cela suffit à mon bonheur et à celui de mon homme " (A, p. 100). Tout au long du roman est en effet relevée la justesse d'Ismène comme dans cet extrait : "Nous arrivons devant une belle porte, Ismène l'ouvre et après les rues fauves et les murs brûlants de la ville, je découvre une pénombre verte et fleurie qu'anime le bruit d'une fontaine. [...] Tout est juste, je dis : / "C'est notre enfance, les couleurs de notre enfance. La tienne fait penser à Jocaste et pourtant comme elle te ressemble” "(A, p. 48).

Racontant ensuite des faits se déroulant avant sa naissance, Ismène conservera ce mode de récit embrayé qui se distingue par son ancrage dans la situation d'énonciation et une volonté accrue de la part du locuteur d'influencer son auditeur ${ }^{\mathbf{2 8}}$. Elle utilisera principalement le passé composé, ce temps du passé intime-

27 Roselyne Orofiamma, «Le travail de la narration dans le récit de vie », dans Christophe Niewiadomski et Guy de Villers (dir.), Souci et soin de soi. Liens et frontières entre histoire de vie, psychothérapie et psychanalyse, Paris, L'Harmattan, 2002, p. 163-191, ici : p. 170.

28 Je me réfère ici aux travaux sur l'énonciation d'Émile Benveniste, notamment "Les relations de temps 
ment lié à la situation d'énonciation et présenté parfois comme l'« accompli du présent ». L'utilisation d'un même régime temporel pour décrire la faute maternelle et sa perpétuation au moment du récit instaure un sentiment de continuité, un effet que n'aurait pu obtenir le passé simple : "Nous avons tous aimé le soleil de Jocaste mais il avait en elle un autre astre " et plus loin "Le malheur a été grand " (A, p. 107). Ismène rapporte pour une bonne partie du récit les propos d'Eudoxia. Cette mise en scène de la nourrice dans la scène de conte n'est pas anodine. Elle exhibe le caractère de reconstitution, dont il s'agit toujours par ailleurs dans un récit sur la petite enfance. Comme dans les récits de vie pratiqués en cure, l'enjeu n'est pas " l'actualisation d'un passé, mais un construit qui s'énonce dans un présent et dans une interaction dont il tire sa signification " ${ }^{29}$. Les récits d'Ismène et les gestes concomitants d'Antigone sont autant d'étapes sur le chemin d'une prise de connaissance conjointe. La performativité du langage, la résistance du sujet, sa détresse émotionnelle mais aussi l'écoute attentive et non interventionniste de l'auditeur et le lien qui les unit sont autant d'indices qui font signe vers la pratique psychanalytique. À l'image des statues de Jocaste se dessine toutefois une double perspective. Parallèlement à la construction de l'identité énonciative d'Ismène s'esquisse celle du sujet à l'écoute. Le chapitre met en effet en exergue la force de l'écoute active d'Antigone et sa potentialité créatrice, à l'instar des figures de thérapeutes présentes dans d'autres textes de l'auteur.

\section{Un monologue adressé}

Ce monologue comporte une dimension dialogique très forte. Au contraire du regard, disqualifié tout au long du récit de création, la voix est " toujours et irrémédiablement "relationnelle" ", comme le rappelle Chiara Elefante. Le discours prononcé engage donc, contrairement à la vision, "une réciprocité entre celui qui le produit et celui qui le perçoit ; il postule donc pour être reçu la nécessité d'une écoute ${ }^{\mathbf{3 0}}$. La relationalité imprimée à la voix d'Ismène, le désir d'aller vers l'autre en partant de soi, trouve un écho chez son auditrice sous une modalité particulière de discours. Antigone ponctue la narration de sa sœur par " et alors ? ", repris bientôt par Ismène. Cette expression remplit une fonction " phatique " qui sert à maintenir vive la communication et met l'accent sur le lien entre les interlocuteurs plutôt que sur l'information transmise, comme dans cet exemple : " Je m'arrête, je n'ai plus envie de continuer, Antigone, mais tes mains travaillent le bois sans hâte et sans arrêt et tu me demandes de la même voix aigue : "Et alors ?" Je soupire, tu me forces à parler "(A, p. 111). Les redoublements thématiques entre les deux chapitres invitent le lecteur à considérer conjointement les pratiques créatrices, comme

\footnotetext{
dans le verbe français ", dans Problèmes de linguistique générale I, Paris, Gallimard, 1966, p. 237, et d'une façon plus générale à ceux de Roman Jakobson, Essai de linguistique générale, Paris, Minuit, 1963.

29 Roselyne Orofiamma, art. cit., p. 172.

30 Adriana Cavarero, citée par Chiara Elefante, "La voix du corps, la voix féminine dans l'œuvre narrative d'Henry Bauchau ", dans Catherine Mayaux et Myriam Watthee-Delmotte (dir.), Henry Bauchau. Écrire pour habiter le monde, Saint-Denis, op.cit., p. 147-160, ici p. 54.
} 
la critique l'a déjà montré. Le geste d'Antigone reste suspendu au récit d'Ismène au flot de ses paroles, rappelant que " la voix non seulement annonce la relation, mais l'annonce en tant qu'instrument matériel, corporel, enraciné dans la singularité d'un individu qui, à travers son souffle, appelle et convoque l'autre à l'écoute „31. Ismène n'entre pas de plein fouet dans la logique articulée de la narration mais commence par « s'agite[r] et bourdonne[r] éperdument » (A, p. 103), afin de saturer l'espace auditif. Cet élément répond d'ailleurs à la sonorité d'Ismène relevée dans l'ensemble du roman : "Et il sort car il entend arriver Ismène ", "J'entends quelqu'un qui franchit l'entrée du jardin, j'espère que c'est Hémon mais ce n'est pas son pas ", etc. - A, p. 51 et 99, pour les exemples donnés) Dans la suite du monologue, on assiste au lent dévoilement d'une vérité complexe et douloureuse, grâce à l'utilisation de plusieurs types de langages artistiques et points de vue. Son résultat, la dualité de la mère et l'inégalité subséquente, semble au contraire être compris de façon univoque. Cette reconnaissance du passé qui passe par son acceptation intime va permettre d'agir sur le présent : "J'aimais Étéocle mais comme notre mère je l'aimais mal, dans la perpétuelle comparaison avec Polynice. [...] Grâce à toi j'ai senti dans mes mains et dans tout mon être une proximité, une compassion pour celui qui, comme moi, a toujours dû prendre le chemin le plus long "(A, p. 121).

Il s'agit donc d'une vérité qui, si elle peut être partagée, est toujours modalisée, puisqu'elle est le fait de la mise en discours par un sujet. Elle naît donc de l'entreprise de réappropriation énonciative d'Ismène et de sa manière singulière " de fluer "32. C'est bien son rythme, en tant qu'" organisation [...] de la subjectivité et de la spécificité d'un discours " (pour le dire avec Meschonnic ${ }^{33}$ ), qui fait revivre le passé familial. Il le réorganise pour lui donner un sens, ce qui permet de mettre à distance la figure de Jocaste et ses défaillances. Le roman, en se donnant le temps d'être à l'écoute d'Ismène et de ses préoccupations quotidiennes, ouvre un nouvel espace de parole qui répond à l'intertexte principal sophocléen mais aussi à toute une tradition de (r)écritures. Chez Henry Bauchau, Ismène se fait le porte-drapeau d'une (r)écriture axée sur le lien familial, la gestion équilibrée du conflit, la mise en avant de personnages secondaires et d'une certaine recherche du bonheur, des préoccupations qui, à première vue, interfèrent avec la représentation courante d'Antigone.

Nadège Coutaz

Université de Lausanne \& Facultés universitaires Saint-Louis (Bruxelles)

31 Ibid, p. 151.

32 Émile Benveniste, "La notion de "rythme" dans son expression linguistique ", dans Problèmes de linguistique générale, op. cit., 1966, p. 327-335.

33 Henri Meschonnic, Poétique du traduire, Lagrasse, Verdier, 1999, p. 99-100. 